\title{
Protect African vultures from poison
}

Antoni Margalida

Darcy Ogada

André Botha

Of the 16 Old World vulture species, $81 \%$ are globally threatened or nearthreatened. Most of these species are declining, particularly in Asia and Africa, mainly due to anthropogenic activities such as the illegal use of poisons. In Africa during the past 30 years, populations of eight vulture species declined by an average of $62 \%$. Ivory poachers are partly to blame. In an act known as sentinel poisoning, poachers use poison to kill elephants or to contaminate their carcasses in an effort to eliminate vultures that, by flying overhead, would reveal the poachers' presence. Deliberate killing of vultures to trade body parts for beliefbased uses (such as traditional medicine) also poses a substantial threat to African vulture populations. From January 2018 to date there have been more than 1000 vultures poisoned in Africa, including incidents in Mozambique, South Africa, Botswana, Zambia, Namibia, Tanzania, and Kenya.

For millennia, vultures have provided ecosystem services by removing animal carcasses and other human waste (thereby controlling diseases), recycling nutrients, and providing cultural inspiration and recreational value. The sustainability of free-ranging vulture populations depends on our ability to identify and mitigate existing and future threats. African governments should prioritize the reduction of illegal poisoning related to traditional medicine and poaching, as well as the education of their citizens about the dangers of pesticide misuse.

Urgent action is needed to harmonize human development in Africa with vulture conservation. The implementation of national and regional vulture conservation strategies, such as the Multispecies Action Plan to Conserve African-Eurasian Vultures (Vulture MsAP), adopted by African and other range countries in October 2017, was an important first step. Although some actions recommended by the Vulture MsAP to reduce the impact of poisoning on the continent's vultures have been implemented in some areas in southern, east, and west Africa, a lot more needs to be done. For example, better coordination of such activities in identified poisoning hotspots is needed. Efforts to curb the impact of poisoning and to establish vulture safe zones are underway in southern and east Africa, but limited action has been taken in elsewhere in Africa. For the plan to succeed, its implementation will also need more substantive material and financial support from African governments. 